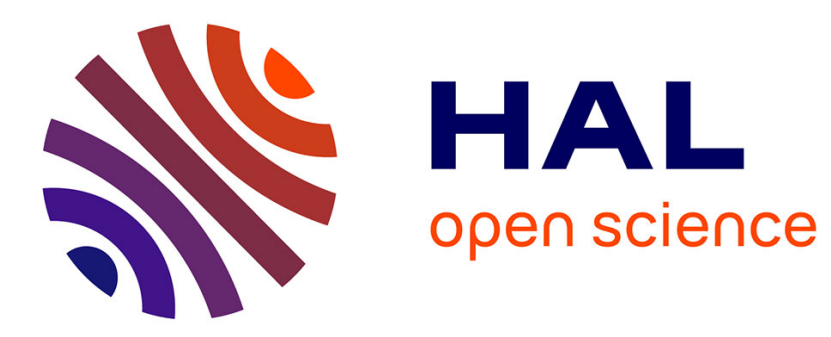

\title{
Parameterized Strategies Specification in Maude
}

\author{
Rubén Rubio, Narciso Martí-Oliet, Isabel Pita, Alberto Verdejo
}

\section{To cite this version:}

Rubén Rubio, Narciso Martí-Oliet, Isabel Pita, Alberto Verdejo. Parameterized Strategies Specification in Maude. 24th International Workshop on Algebraic Development Techniques (WADT), Jul 2018, Egham, United Kingdom. pp.27-44, 10.1007/978-3-030-23220-7_2 . hal-02364577

\section{HAL Id: hal-02364577 \\ https://hal.inria.fr/hal-02364577}

Submitted on 15 Nov 2019

HAL is a multi-disciplinary open access archive for the deposit and dissemination of scientific research documents, whether they are published or not. The documents may come from teaching and research institutions in France or abroad, or from public or private research centers.
L'archive ouverte pluridisciplinaire HAL, est destinée au dépôt et à la diffusion de documents scientifiques de niveau recherche, publiés ou non, émanant des établissements d'enseignement et de recherche français ou étrangers, des laboratoires publics ou privés. 


\title{
Parameterized Strategies Specification in Maude $^{\star}$
}

\author{
Rubén Rubio $\left.^{(}\right)$, Narciso Martí-Oliet, Isabel Pita, Alberto Verdejo \\ Universidad Complutense de Madrid, Spain \\ \{rubenrub, narciso, ipandreu, jalberto\}@ucm.es
}

\begin{abstract}
Strategies and parameterization are two convenient tools for building clear and easily configurable specifications of complex computational systems, compositionally. Parameterization is a widely used feature of the Maude rewriting framework, whose strategy language implementation we have recently completed with strategy modules.

This paper describes the Maude strategy language and the associated parameterization techniques. Then, the specification and analysis of some examples of strategy parameterized systems are shown.
\end{abstract}

Keywords: Rewriting logic $\cdot$ Strategies $\cdot$ Maude $\cdot$ Parameterized specification

\section{Introduction}

Strategies are ubiquitous in Computer Science. As recipes to tackle search problems and bound nondeterminism, they appear in algorithms, automatic deduction, language semantics, artificial intelligence, .... In rewriting logic [13], some of these examples are better specified compositionally, abstracting not only data representation and rules but also the way they are applied. This parametric control of the rewriting process is conveniently expressed using strategies that take other strategies as parameters.

Maude $[6,7]$ is a declarative high-level language based on rewriting logic that allows the description, execution and analysis of concurrent and distributed system models at different levels. First, sorts, symbols, equations and membership axioms are expressed in terms of membership equational logic [3]. Then, we add rewrite rules to represent transitions of a concurrent system, which need neither be deterministic, nor confluent, nor terminating. Above this, we can control how rules are applied using a strategy language [8,11]. Its implementation, at the Core Maude level in $\mathrm{C}++$, has been recently completed as an extension of Maude 2.7.1 [7].

The strategy language is based in its authors experience with strategies in Maude and in previous strategy languages like ELAN [2] and Stratego [5]. It

\footnotetext{
* Research partially supported by MCIU Spanish project TRACES (TIN2015-67522C3-3-R), and by Comunidad de Madrid project N-Greens Software-CM (S2013/ICE2731).
} 
has already been exploited in the specification of algorithms, inference systems, and language semantics: Milner's CCS [12], the ambient calculus [12], the semantics of the parallel functional language Eden [9], equational logic completion procedures [16], a proof calculus for membrane systems [1], etc. These examples are likely to be expressed and generalized using control parameterization with strategies, whose implementation was not available at that time. Once expressed in this way, the specified systems can be both executed and tested with different alternative strategies provided as parameters, or analyzed at different levels with specific tools, like a model checker.

The next section introduces Maude, its strategy language, and the rudiments of parameterization. A generic backtracking scheme serves as an introductory example. The following sections describe some other examples of parameterized systems, targeting the simplex algorithm, the $\lambda$-calculus, and a functional program interpreter. These and more examples can be downloaded from [15], as well as the current version of Maude with full strategy support.

\section{Maude}

A Maude program consists of a hierarchy of modules, describing the data representation and behavior of the system specified. There are different module types for different specification levels.

Functional modules define membership equational logic theories, whose signature $(K, \Sigma, S)$ consists of a set of kinds $K$, a many-kinded collection of operators $\Sigma=\left\{\Sigma_{k_{1} \cdots k_{n}, k}:\left(k_{1} \cdots k_{n}, k\right) \in K^{*} \times K\right\}$, and $S=\left\{S_{k}: k \in K\right\}$ a many-kinded set of partially ordered sorts. Equations and sort membership axioms $E$ are defined on them

$$
(\forall X) \quad \begin{gathered}
t=t^{\prime} \\
t: s
\end{gathered} \text { if } \bigwedge_{i} u_{i}=u_{i}^{\prime} \wedge \bigwedge_{j} v_{j}: s_{j}
$$

In addition, operators can be annotated with structural axioms, like commutativity (comm), associativity (assoc), and identity (id). For example, the following functional module specifies sets of integer numbers using both equations and axioms.

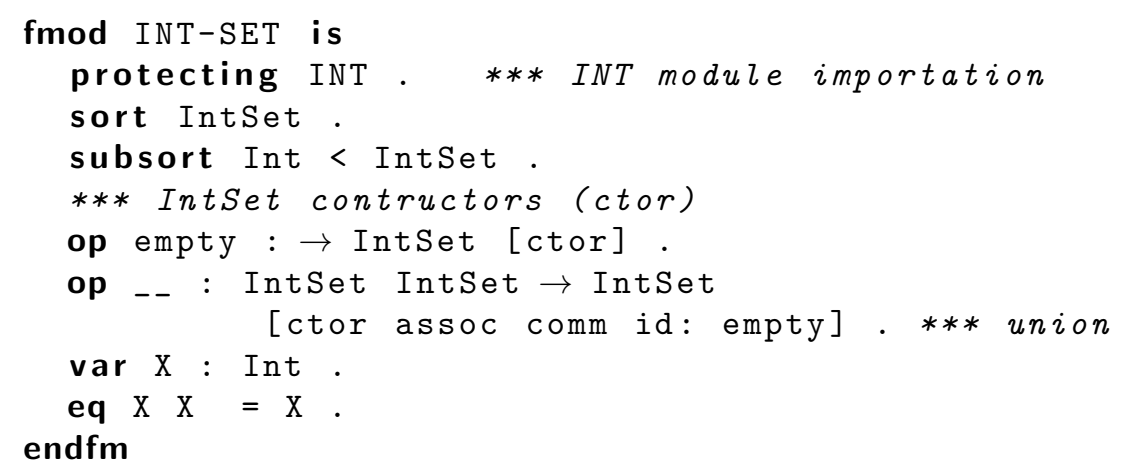


Functional modules are bound to some executability requirements, like confluence, termination, and sort-decreasingness.

System modules describe rewriting logic theories $\mathcal{R}=(\Sigma, E \cup A, R)$, adding rewriting rules $R$ on top of the equational theory. Rules do not have to be either confluent or terminating, so they are likely to express non-deterministic behavior.

$$
(\forall X) \quad t \Rightarrow t^{\prime} \text { if } \bigwedge_{i} u_{i}=u_{i}^{\prime} \wedge \bigwedge_{j} v_{j}: s_{j} \wedge \bigwedge_{k} w_{k} \Rightarrow w_{k}^{\prime}
$$

Anyhow, rules are required to be coherent with equations and axioms [7, §5.3]. Conditions of the third type are called rewriting conditions, and hold true iff the term $w_{k}$ can be rewritten to match the term $w_{k}^{\prime}$.

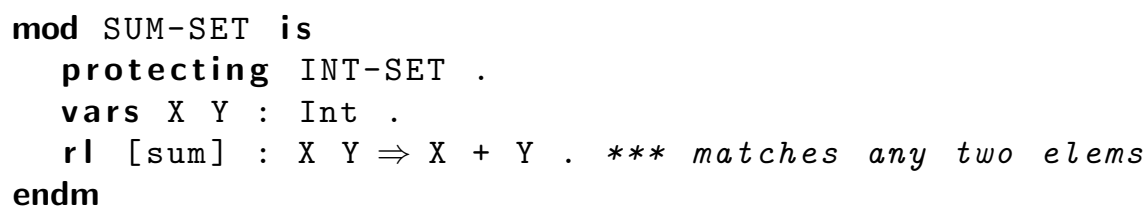

The module SUM-SET above introduces a rule sum that takes two integers in the set and replaces them by their sum.

Strategy modules allow finer control of the rule rewriting process by means of the strategy language and recursive strategy definitions. They are described in the next section.

\subsection{The strategy language}

Rules in rewriting logic can be applied in any order, at any position, and with different matches. Maude provides various commands, like rewrite and frewrite, that execute all available rules against a given term until none can be applied or an optional step bound is reached. They use some internal fixed criteria to select the next rule application. In turn, the search command explores all possible rewriting paths to find a target term meeting some conditions. However, the specifier is sometimes interested in imposing constrains on the allowed execution paths, like a particular precedence of rules, or to which subterms they must be applied, etc. This is when strategies get inside the game.

From the point of view of the results, a strategy $\alpha$ is an operation transforming a term $t$ into a set of terms, since the restrictions need not make the process deterministic. Strategies can be executed with the command srewrite $t$ using $\alpha$. The most elementary strategy is rule application

$$
\text { top (label } \left.\left[x_{1}<-t_{1}, \ldots, x_{n}<-t_{n}\right]\left\{\alpha_{1}, \ldots, \alpha_{m}\right\}\right) \text {, }
$$

that executes any available rules with label label on any subterm of the subject term. Variables in the rule and its condition can be instantiated before application with the substitution that maps $x_{k}$ to $t_{k}$, and if the rule contains any rewriting condition, it must be controlled with a substrategy $\alpha_{k}$. Moreover, the 
optional top modifier restricts the application of the rule to the top of the subject term. A more powerful tool for selecting where to apply a strategy is the matchrew operator

matchrew $P\left(x_{1}, \ldots, x_{n}\right)$ s.t. $C$ by $x_{1}$ using $\alpha_{1}, \ldots, x_{n}$ using $\alpha_{n}$

It matches the pattern $P$ on top of the subject term, and for each match satisfying the condition $C$, the subterms corresponding to $x_{1}, \ldots, x_{n}$ are rewritten using $\alpha_{1}, \ldots, \alpha_{n}$, and reassembled again. The operator name can be prefixed by a or $\mathrm{x}$ to match anywhere within the term or modulo structural axioms. Similar format follow the tests match $P$ s.t. $C$, to check if $P$ matches the subject term and satisfies $C$. Regular expressions are included in the strategy language by means of the alternation $\alpha \mid \beta$, the concatenation $\alpha$; $\beta$, the Kleene star $\alpha^{*}$, and the constants idle and fail. A conditional strategy $\alpha ? \beta: \gamma$ is also available. It executes $\alpha$ and then $\beta$ on its results, but if $\alpha$ does not produce any, it applies $\gamma$ to the initial term.

The last ingredient of the strategy language are potentially recursive named strategies. These strategies are defined in strategy modules, which have the form smod $M$ is ... endsm and contain:

- Strategy declarations strat sname : T1 .. Tn @ T, which state their parameter types $T_{1}$ to $T_{n}$, and the type $T$ of the subject terms the strategy will be applied to.

- Strategy definitions sd sname $\left(t_{1}, \ldots, t_{n}\right):=\alpha$. The free variables in the right-hand side strategy expression $\alpha$ must be included in the terms $t_{1}$ to $t_{n}$. Conditional strategy definitions, introduced by csd, are available too, and impose conditions as in regular equations. A named strategy can be given any number of strategy definitions, and all definitions whose left-hand side matches the call term will be executed.

Now, we will illustrate all the different strategy constructors described above with a very simple example. Consider the following system module:

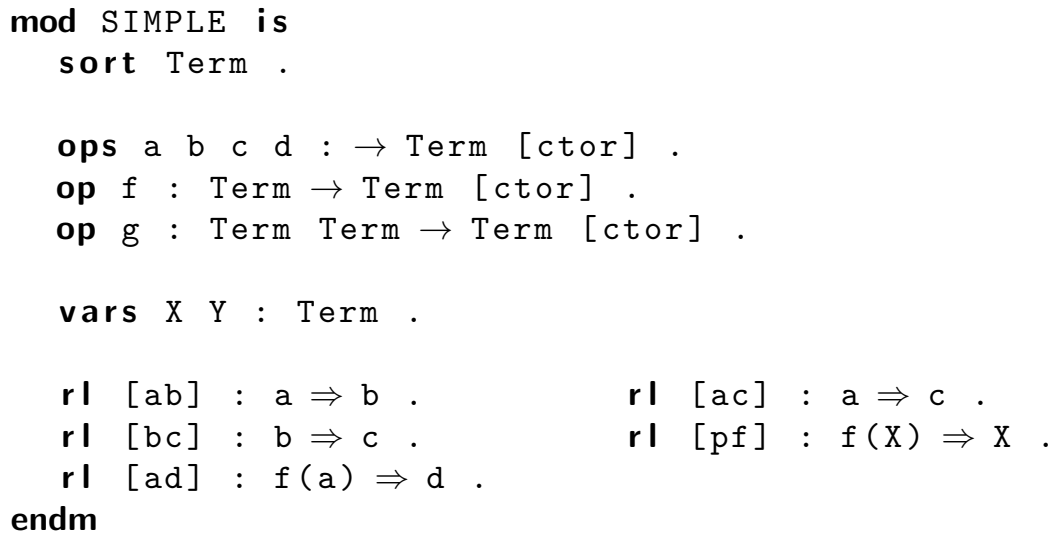


The SIMPLE module defines some constants and functions to build terms, and various rules that transform them. For example, we can apply the pf rule to the term $f(g(f(a), b))$ by means of the srewrite command, whose initial keyword can be abbreviated to srew:

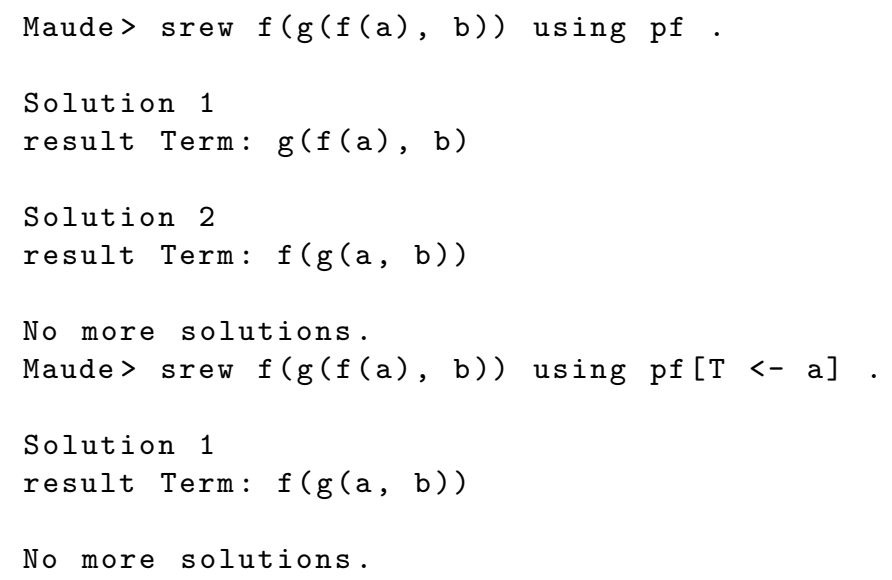

Notice that all possible rewrites are returned as different solutions. In the first case, the rule can be applied both to the first and to the second occurrence of $f$. However, in the second case, the initial substitution restricts its application to the subterm $f(a)$. Using the strategy top(pf) instead, pf is only applied on top and only $g(f(a), b)$ is obtained. Finer precision on where to apply a strategy can be achieved by the subterm rewriting operator. For example, matchrew $\mathrm{g}(\mathrm{X}, \mathrm{Y})$ by $\mathrm{X}$ using pf restricts the application to the first argument of $g$. Then $g(f(b), f(c))$ rewrites only to $g(b, f(c))$. Moreover, multiple subterms can be rewritten in parallel by strategies like

amatchrew $\mathrm{g}(\mathrm{X}, \mathrm{Y})$ by $\mathrm{X}$ using $\mathrm{pf}, \mathrm{Y}$ using $\mathrm{bc}$, which transforms the initial term $f(g(f(a), b))$ in $f(g(a, c))$.

Once we are able to apply strategies to particular subterms, we need regular expressions to compose rewriting sequences. If we rewrite $\mathrm{g}(\mathrm{a}, \mathrm{b})$ by the concatenation $\mathrm{ab} ; \mathrm{bc}$, we obtain $\mathrm{g}(\mathrm{c}, \mathrm{b})$ and $\mathrm{g}(\mathrm{b}, \mathrm{c})$, since the rule $\mathrm{bc}$ has been applied after $\mathrm{ab}$, at the same or at a different position. Instead, when executing the alternation $a b \mid \mathrm{bc}$, only one of these is applied, and we get $\mathrm{g}(\mathrm{b}, \mathrm{b})$ and $\mathrm{g}(\mathrm{a}, \mathrm{c})$ as solutions. Finally, the iteration pf* applies pf zero or more times consecutively, so it rewrites $f(f(a))$ to $f(f(a)), f(a)$, and a.

Other useful resources are the tests like match $\mathrm{g}(\mathrm{X}, \mathrm{Y})$ s.t. $\mathrm{X} \neq \mathrm{Y}$. It does not produce any solution for $\mathrm{g}(\mathrm{a}, \mathrm{a})$, but for $\mathrm{g}(\mathrm{a}, \mathrm{b})$, the solution is the term itself. Tests easily combine with conditionals. For example, the strategy match $f(X)$ ? ab : bc applies ab if the subject term top symbol is an $f$, and otherwise executes bc. Then, $f(a)$ rewrites to $f(b), b$ to $c$, and no solution is produced for a. However, conditionals can be used with any strategy as condition, as we will see soon and throughout the paper.

Finally, to define named and recursive strategies, we need to write strategy modules like the following: 


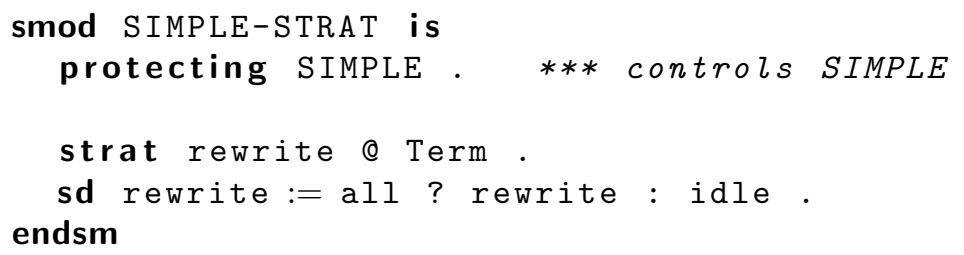

where all is a built-in constant that executes any available rule. In this case, it can be seen as equivalent to ab | ac | bc | pf | ad. Hence, the strategy rewrite applies any rule until no more rules can be applied, as the usual rewrite command does. Running this strategy for the term $g(f(a), f(b))$ we obtain:

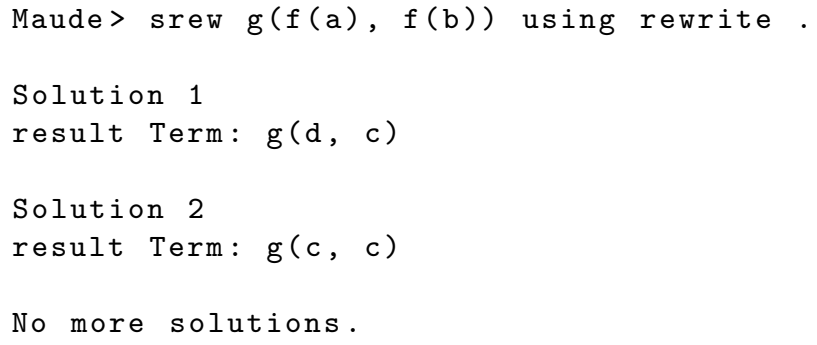

In Figure 1, the strategy language semantics is described in brief. Apart from the initial term, the semantic function $\llbracket \alpha \rrbracket:\left(X \rightarrow T_{\Sigma / E}\right) \times T_{\Sigma / E} \rightarrow \mathcal{P}\left(T_{\Sigma / E}\right)$ receives a substitution by way of variable environment, because function calls and matchrews bind variables. The semantics of the strategy definitions $\delta$ is calculated as a fixed point of a continuous operator, and here $\Delta\left(s l, t_{1} \cdots t_{n}\right)$ refers to the set of strategy definitions for $s l$ whose left-hand side matches $t_{1}, \ldots, t_{n}$, along with the corresponding substitution $\sigma$.

\subsection{Parameterization}

Parameterization is achieved using three basic building blocks: theories, views, and parameterized modules [6,7]. A parameterized module is a usual module taking a set of formal parameters, bound to some theories.

$\bmod \operatorname{NAME}\{\mathrm{X} 1:: \mathrm{TH} 1, \ldots, \mathrm{XN}:: \mathrm{THN}\}$ is $\ldots$ endm.

A theory declares the interface, the syntactic and semantic requirements, the actual parameter must respect. Each module type (functional, system, strategy) has its theory counterpart. They are structurally identical, but theories are not required to fulfill the executability properties of a module. Module parameters can only be bound to theories of their type or simpler types. Finally, a view is the way to express how a module honors a theory, mapping each sort, operator, and strategy declared in the theory to the actual value in the module. Then, views are used to instantiate parameterized modules.

As an example, the most basic predefined theory is TRIV, which declares a single sort. 


$$
\begin{aligned}
& \llbracket \text { idle } \rrbracket(\theta, t)=\{t\} \\
& \llbracket \text { fail } \rrbracket(\theta, t)=\emptyset \\
& \llbracket \alpha ; \beta \rrbracket(\theta, t)=\bigcup_{t^{\prime} \in \llbracket \alpha \rrbracket(\theta, t)} \llbracket \beta \rrbracket\left(\theta, t^{\prime}\right) \quad \llbracket \alpha \mid \beta \rrbracket(\theta, t)=\llbracket \alpha \rrbracket(\theta, t) \cup \llbracket \beta \rrbracket(\theta, t) \\
& \llbracket \alpha * \rrbracket(\theta, t)=\bigcup_{n \geq 0} \llbracket \alpha \rrbracket^{n}(\theta, t) \\
& \llbracket \alpha ? \beta: \gamma \rrbracket(t)= \begin{cases}\llbracket \alpha ; \beta \rrbracket(\theta, t) & \text { if } \llbracket \alpha \rrbracket(\theta, t) \neq \emptyset \\
\llbracket \gamma \rrbracket(\theta, t) & \text { if } \llbracket \alpha \rrbracket(\theta, t)=\emptyset\end{cases} \\
& \llbracket \text { match } P \text { s.t. } C \rrbracket(\theta, t)= \begin{cases}\{t\} & \text { if } \operatorname{match}\left(P, t, C, \theta_{-\operatorname{occur}(P)}\right) \neq \emptyset \\
\emptyset & \text { otherwise }\end{cases}
\end{aligned}
$$

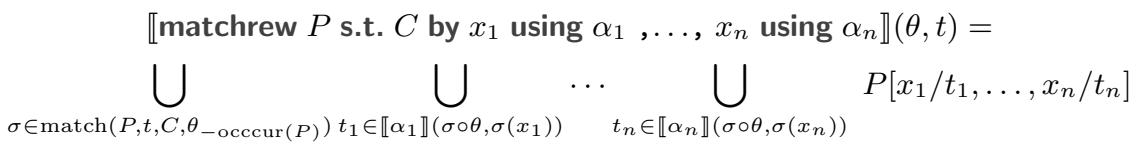

$$
\begin{aligned}
& \llbracket s l\left(t_{1}, \ldots, t_{n}\right) \rrbracket(\theta, t)=\bigcup_{(\delta, \sigma) \in \Delta\left(s l, \theta\left(t_{1}\right) \cdots \theta\left(t_{n}\right)\right)} \llbracket \delta \rrbracket(\sigma, t)
\end{aligned}
$$

Fig. 1. Strategic set-theoretic semantics definitions

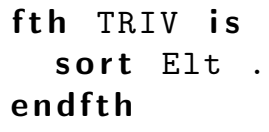

The module NAT can be viewed as a TRIV:

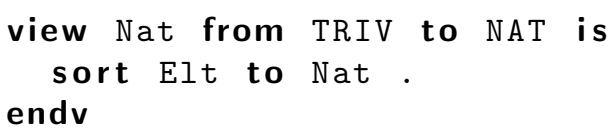

Then, the view Nat can be used to instantiate modules like lists LIST $\{$ Nat\}, sets SET $\{$ Nat $\}, . .$. Module instantiation is based on the pushout along a view:

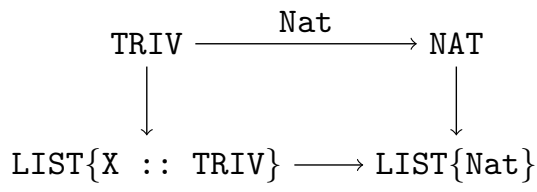

A simplified version of the LIST module is the following:

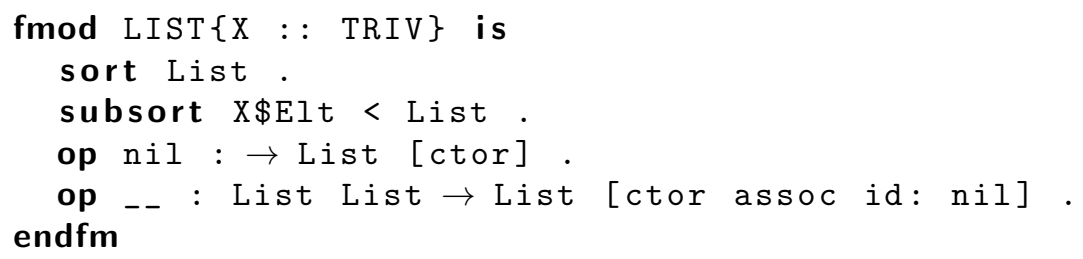


Inside parameterized modules, sorts coming from theories are accessed by prefixing their names by their parameter name followed by a $\$$ sign. Operators and strategies retain their names as in the parameter theory. The Maude standard prelude includes some general theories like TRIV, TOTAL-PREORDER, STRICT-WEAK-ORDER, . . , and many views from the standard data types to those theories $[6,7]$.

\section{$3 \quad$ An introductory example}

A simple example of parameterized control is the generic backtracking scheme.

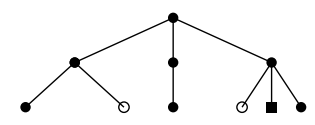

The abstract backtracking problem is specified in two nested theories, the first proclaims the functional requirements, and the second extends it with the strategic ones. They require a State sort for states, and a strategy expand to rewrite a state to any of its direct successors, non-deterministically. We admit that the generated successors may not be valid, so we require a predicate is $0 \mathrm{k}$ to test them, and also a predicate isSolution to determine whether a given state is already a solution.

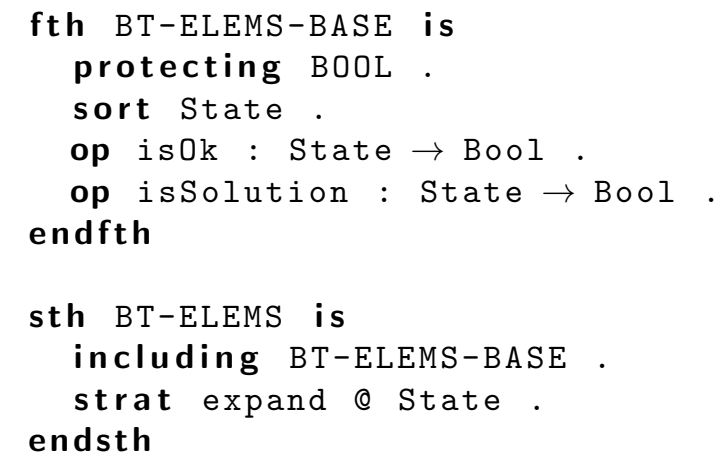

Then, a parameterized module BT-STRAT, given the specification of the problem following BT-ELEMS, defines a strategy solve for executing the backtracking algorithm. This is the following module, where parameters are highlighted in italics.

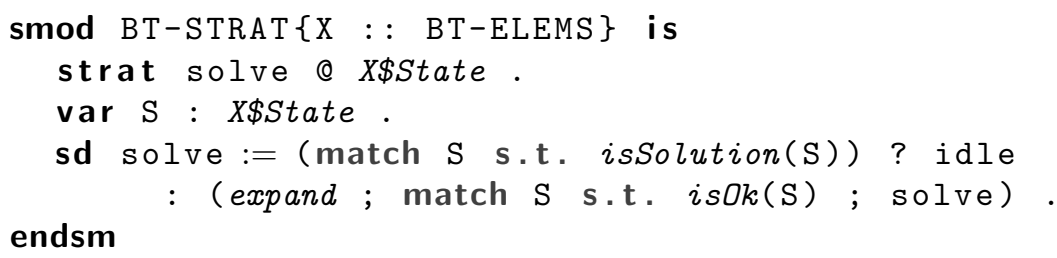

The solve strategy is recursive. It concludes successfully when it finds a solution. Otherwise, it applies the strategy expand to obtain a successor, tests 
whether it is valid, and iterates the process by a recursive call. Rewriting paths are discarded either when a successor not satisfying is $0 \mathrm{k}$ is reached or when the expand strategy does not provide any successor. Indeed, each result of the expand strategy opens a new branch of the execution tree.

Although we call it backtracking, the strategy is abstract enough to be interpreted or executed otherwise, depending on the order in which the branches are explored. For backtracking, we understand a depth-first exploration, going backwards to try another branch when the current one has been exhausted without finding a solution. However, the default strategy rewriting implementation obeys a fair scheduling, which combines depth and breadth-first search. Here, the fair execution policy will usually require more time and memory usage than a real backtracking.

The generic algorithm is useless without actual instances. They can be specified in separate modules or files, but finally they have to fit in the frame of an abstract backtracking problem by means of a view from BT-ELEMS. The following module specifies the search for a Hamiltonian cycle in a graph, i.e. a cycle visiting every vertex of the graph only once.

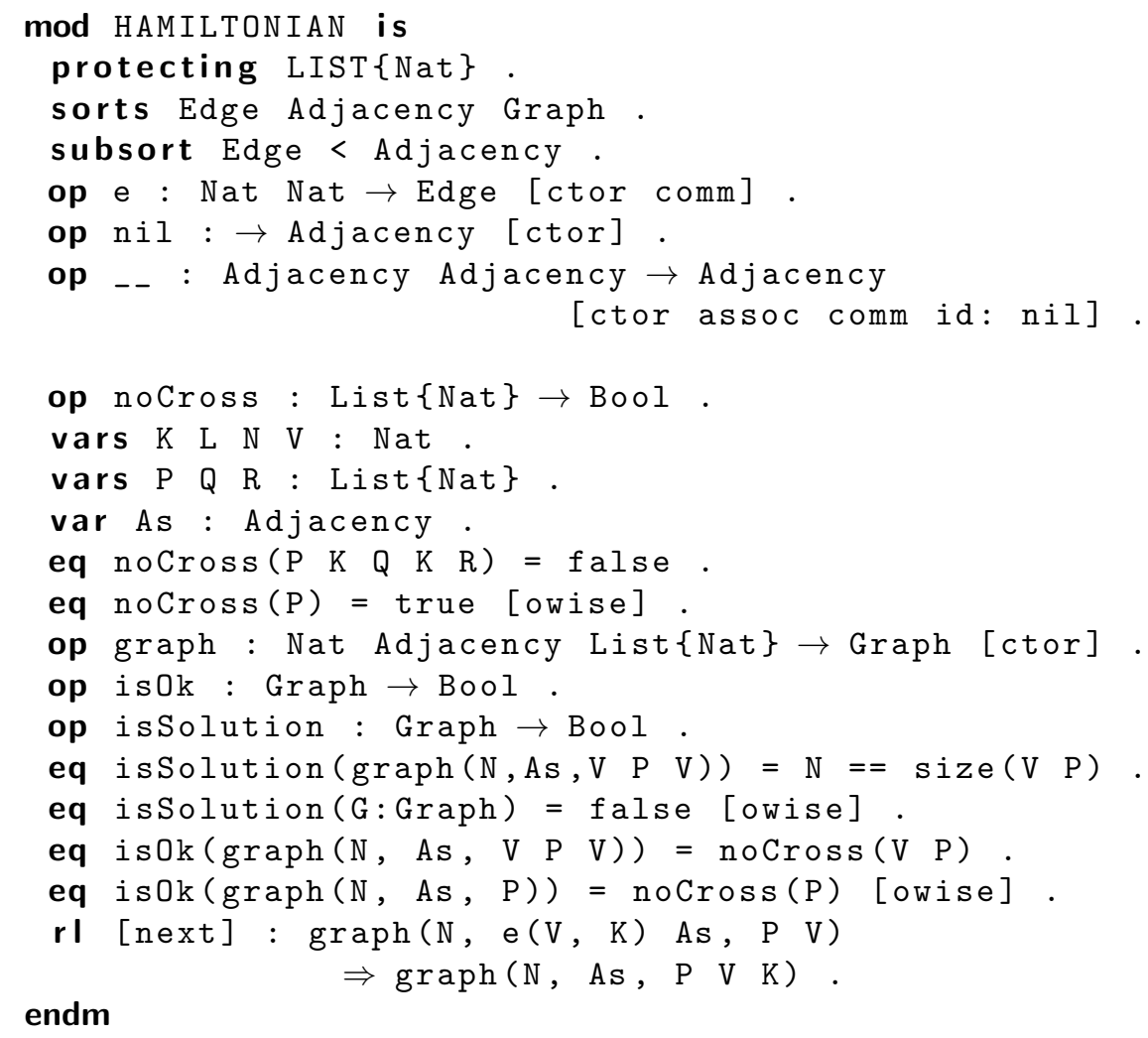

The module defines Graph, including both the graph and the current path, which can be extended up to a Hamiltonian cycle using the rule next whenever possible. Finally, as we said, the problem has to be presented as a backtracking 
instance using a view. Identity mappings do not need to be written, but we have included them to illustrate the syntax.

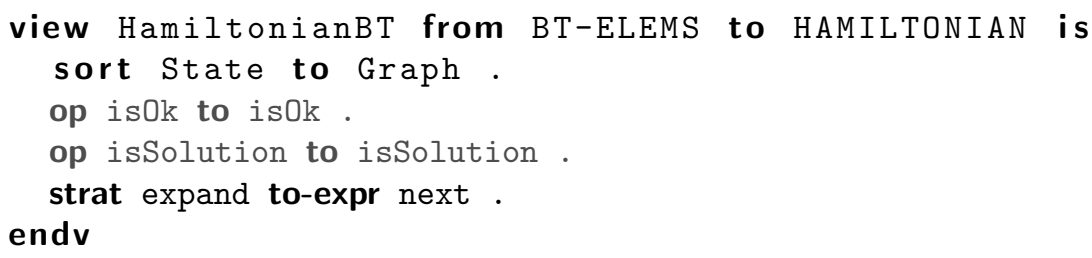

This instance is specially simple, the expand strategy has been defined inline as the next rule. In general, more elaborated strategies can be defined in a strategy module. In that case, the strategy module must be the to part of the view and the strategy is mapped by strat expand to sname. This can be seen in other examples available in the webpage [15] like the labyrinth escape problem, the $n$-queens problem, the graph $m$-coloring problem, etc.

A refinement of backtracking is branch and bound. This algorithmic technique was also programmed with parameterized strategies. Its problem specification, its theories, include richer functional and strategic requirements, so we do not describe them here. In this case, we cannot use an expand strategy that nondeterministically evolves to a successor, we need to examine them all to decide which to explore first according to the rank function. Hence, we have experimented with multiple approaches using different problem signatures. These can also be downloaded from [15].

\section{The simplex algorithm}

The simplex method [14] is a well-known algorithm for solving linear programming problems, i.e. for finding solutions that maximize (or minimize) a linear functional subject to some linear constraints.

$$
\begin{array}{ll}
\max / \min & c_{1} x_{1}+\cdots+c_{n} x_{n} \\
& a_{11} x_{1}+\cdots+a_{1 n} x_{n} \leq b_{1} \\
& a_{21} x_{1}+\cdots+a_{2 n} x_{n}=b_{2} \\
& a_{31} x_{1}+\cdots+a_{3 n} x_{n} \geq b_{3} \\
& x_{1}, \ldots, x_{n} \geq 0
\end{array}
$$

Formulated by George Dantzig in the late 1940s, it has had many industrial applications. Here we present an executable linear programming solver written in Maude using this method. The various non-deterministic steps are controlled by strategies, which are configurable through parameterization.

Figure 2 shows all the specification modules. LINPROG includes some basic definitions of linear algebra (polynomials, inequalities, ..., and operations on them), as well as linear programming problems. Then, SIMPLEX-TABLE defines the simplex tables (the state of the algorithm) and some operations to obtain information and modify them. At the rule level, SIMPLEX-CONSTR defines how 


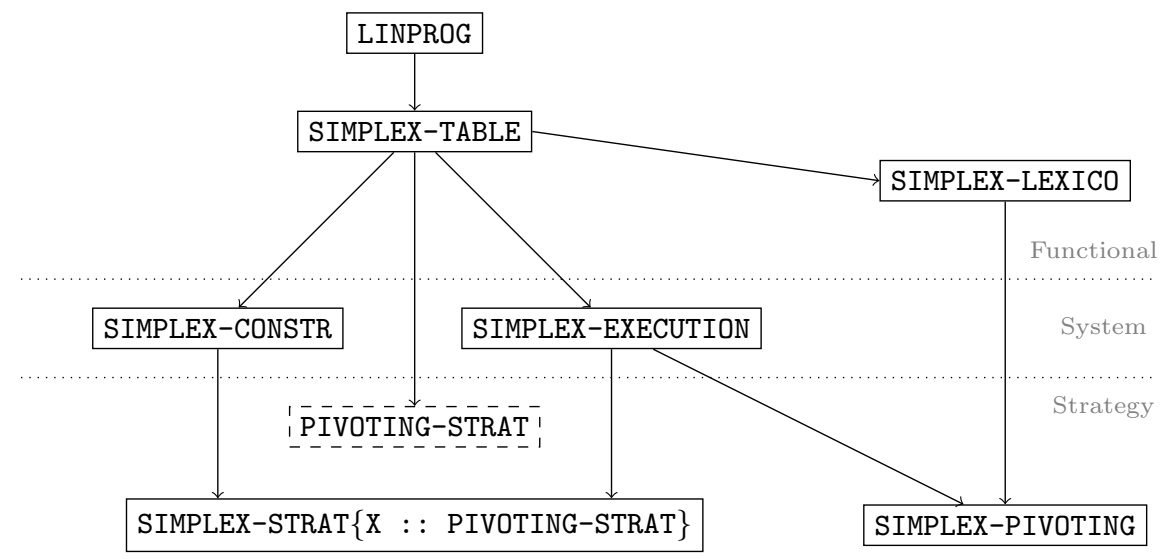

Fig. 2. Module structure for the simplex algorithm specification

to build a table from a linear programming problem, and SIMPLEX-EXECUTION provides the rules for the different actions of the simplex method. The most important is pivot, which changes the algorithm basis

$$
\begin{aligned}
\text { crl [pivot]: } & \text { Table } \Rightarrow \text { pivot }(T a b l e, V e, V l) \text { if } \\
& \text { Ve, } R:=\text { enterVars }(T a b l e) \wedge \\
& V l, S:=\text { leaveVars }(T a b l e, V e) .
\end{aligned}
$$

This rule is non-deterministic because Ve and Vl can be chosen among different alternatives. Pivoting carelessly may even lead to cycles, so that the algorithm may not terminate. Fortunately, there are different cycle prevention techniques to avoid non-termination. The more common ones are the Bland rule and the lexicographic rule. These rules (or any other) can be switched by means of parameterization.

The global solving process starts with a linear programming problem. The first stage is the generation of the simplex table, a non-deterministic process which produces equivalent tables up to renaming. So, we will concentrate on the second stage, the simplex method itself.

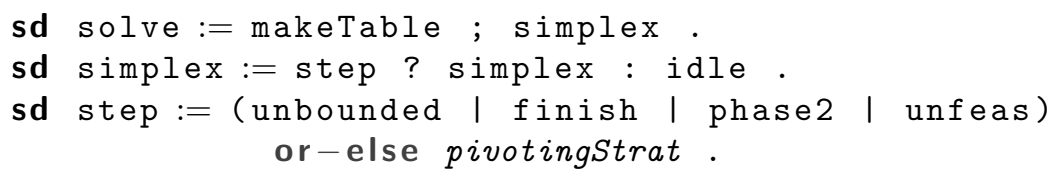

The simplex strategy executes a simplex step until no more can be applied. Each step first applies some rules that transform the table in some specific situations: unbounded detects when the problem is unbounded and transforms the table in a description of the beam of infinite improvement, and finish detects when the problem is already solved and presents the solution in a more readable form. The other rules are related to the two phases method, which may be sometimes needed to find an initial feasible solution of the linear system: unfeas signals 
that the problem is unfeasible; and phase 2 transforms the table to a usual table whenever possible. Finally, if none of these (cheaper and concluding) rules can be applied, the pivoting strategy pivotingStrat is applied. This is the parameter of the SIMPLEX-STRAT module, provided by the theory PIVOTING-STRAT, which declares a single strategy without parameters applicable to the simplex table sort. For instance, the Bland rule is specified in SIMPLEX-PIVOTING with the following strategy

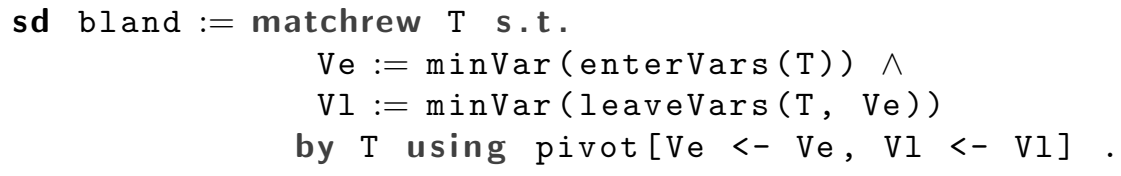

where minVar equationally computes the minimum variable within a set (a total order < is defined on them). A view Bland from PIVOTING-STRAT to SIMPLEX-PIVOTING, mapping pivotingStrat to bland, is then used to instantiate SIMPLEX-STRAT.

\subsection{Parameterized analysis}

A natural analysis of parameterized systems is the comparison of the behavior of their multiple instances, which can be executed or simulated, looking at the execution time, the number of rewriting steps, or the memory requirements. More essential properties can be inspected by tracing the state of the system while running the simulation. Strategies are a useful tool for that purpose. Apart from this, parameterized modules can be analyzed with specific tools like the Maude model checker.

For the simplex method, we have compared the performance of two pivoting strategies and free rewriting in terms of time and number of rewrites, against a linear programming exercises set. Since these properties depend on the actual Maude implementation, we also consider an intrinsic property of the algorithm: the number of iterations or pivoting steps until a solution is found. To obtain this attribute, we count the number of pivot rule executions with the aid of a parameterized analyzer module that maintains a pair watch $(\mathrm{T}, \mathrm{N})$ of a simplex table and a counter, applies the rules to the table using matchrew, and updates the counter accordingly. This can be compared with the unrestricted better case and worst case number of iterations, which can be calculated using strategies too.

\begin{tabular}{|c|c|c|c|}
\hline & Free & Bland & Lexicographic \\
\hline Iterations above better case & 2.05 & 2.05 & 1.47 \\
\hline Number of rewrites & 4246 & 5195 & 5191 \\
\hline Time (ms) & 1.84 & 2.29 & 2.2 \\
\hline
\end{tabular}

We observe that the lexicographic rule reduces the mean number of iterations, but since its decisions require more computations, its performance is similar to Bland's. The free strategy, executing the rules at the discretion of the Maude rewriting engine, performs quite well. 
Regarding auxiliary tools, we have applied an experimental prototype of a strategy-aware model checker. Thanks to it, we can check whether the algorithm following a given strategy, for a fixed example, may cycle or not. Predictably, no example cycles with the Bland and lexicographic rules, but some do with free rewriting.

\section{The $\lambda$-calculus}

The $\lambda$-calculus can be easily expressed and executed in Maude [10]. $\beta$-reduction is the single rule of the rewriting system, so a term can be reduced by simply using the rewrite and search commands.

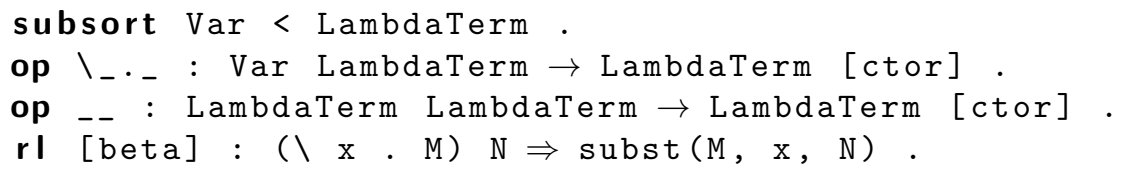

However, which $\beta$-redex is reduced first is an important choice. Some reduction paths may not terminate while others reach an irreducible term, even though this is unique by the Church-Rosser property. The normalization theorem says that reducing the leftmost outermost redex first guarantees finding a normal form in case it exists.

$$
\begin{array}{rl}
(\boldsymbol{K} \boldsymbol{I}) \Omega \longrightarrow(\boldsymbol{K I}) \Omega \curvearrowright & \boldsymbol{K}=\lambda x \cdot(\lambda y \cdot x) \\
(\lambda y . \boldsymbol{I}) \Omega \longrightarrow \boldsymbol{I} & \boldsymbol{I}=\lambda x \cdot x \\
& \Omega=(\lambda x . x x)(\lambda x . x x)
\end{array}
$$

We allow selecting which strategy step to use for a single reduction step and, using a parameterized module, we define a strategy that reduces the term to an irreducible form, if any.

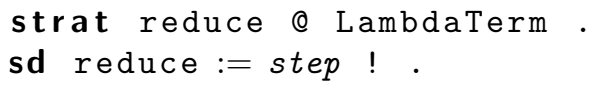

The operator $\alpha$ ! executes $\alpha$ until it cannot be further applied. The different implemented strategies for a single reduction are:

- Applicative order (inner rightmost redex first)

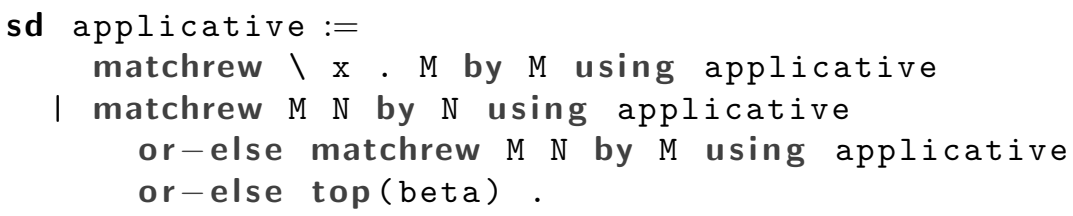

- Normalizing strategy (outer leftmost redex first)

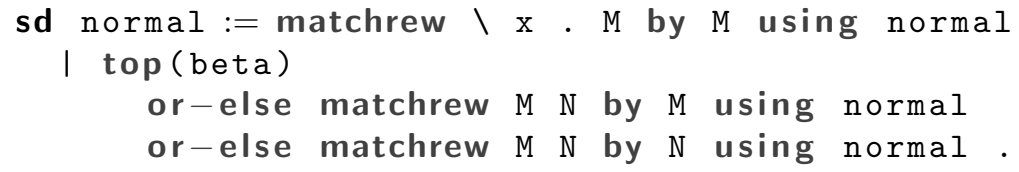


- By name (normalizing but no reduction inside abstraction)

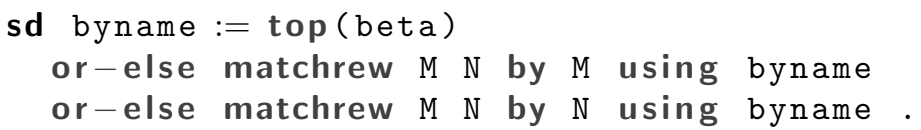

- By value (only outermost redex and when argument is value)

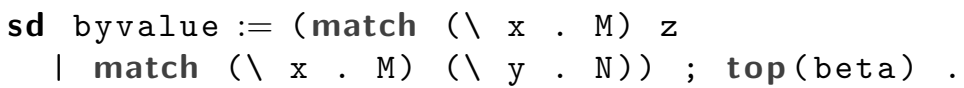

For example, srew (K I) Omega using applicative does not finish, but srew (K I) Omega using normal produces $\backslash \mathrm{x}$. $\mathrm{x}$ as a result. Moreover, depending on the reduction strategy, we obtain different canonical forms. From ( $K$ z $)$ t we get $z$ using all strategies but byvalue, whose canonical form is the term unchanged.

\section{Semantics of programming languages}

The semantics of programming languages is an interesting field to apply both strategies [4] and parameterization. The Maude implementation of Eden [9], a concurrent language based on Haskell, can be cited as example. The general idea is that strategies control the execution process and can be used to tweak some semantic choices, and see how alternatives perform. Here we describe a simpler example, the Recursion equations (REC) language [17, Chapter 9].

A REC program consists of a closed integer expression to evaluate and a set of integer function definitions of the form $f\left(x_{1}, \ldots, x_{a_{f}}\right)=\langle$ expr $\rangle$. Expressions contain integer constants, sums, products, subtractions, conditionals, and calls to any defined function. Hence, functions can be recursive and mutually recursive. An organized set of modules (see Figure 3) specifies the representation of REC programs and its basic rules:

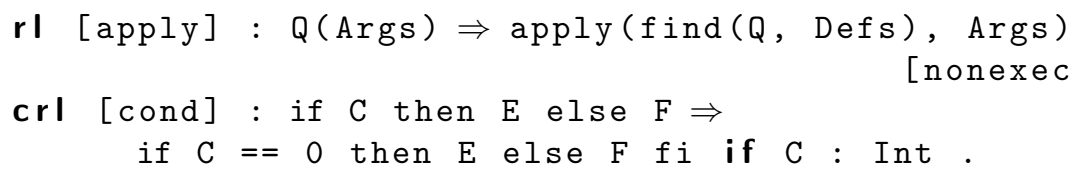

The rule apply replaces a function call Q(Args) by its definition according to the list of definitions Defs, substituting its variables by the call arguments using the equational function apply. This rule is not directly executable since Defs, absent in the left-hand side, must be provided from the context in the application strategy substitution, as we will see. Rule cond resolves a conditional when its condition expression has already been reduced to an integer. In any other case, integer expressions are reduced equationally, since the REC expressions sort RecExpr has been defined as an extension of the built-in sort Int. REC programs are executed following a reduce strategy that receives a list of function definitions as an argument. It is parameterized by a strategy st that is intended to expand function calls, and which we will later instantiate with byvalue and byname alternatives. 


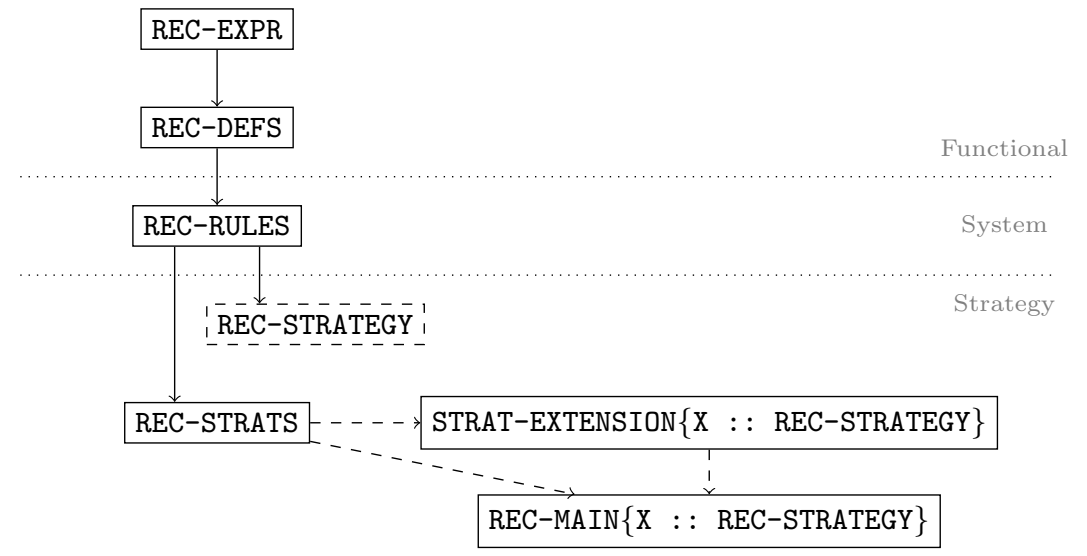

Fig. 3. Module structure for the REC language specification

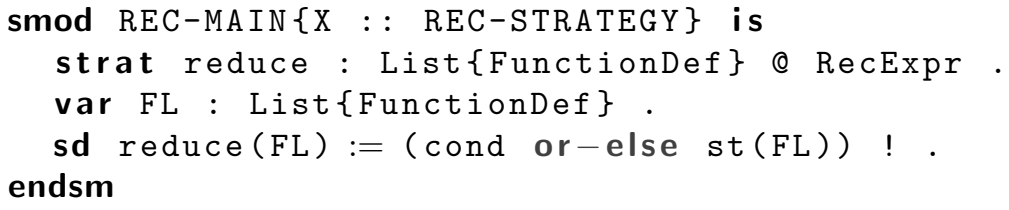

According to the reduce definition, conditionals and calls are reduced as long as possible, but conditionals are reduced first. This is convenient, since function calls may appear anywhere inside the integer expression, and a simple recursive example like the factorial

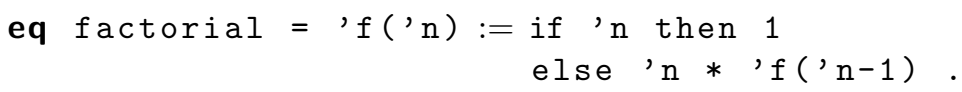

shows that expanding calls anywhere is problematic. In effect, the precedence of cond avoids the non-terminating reduction

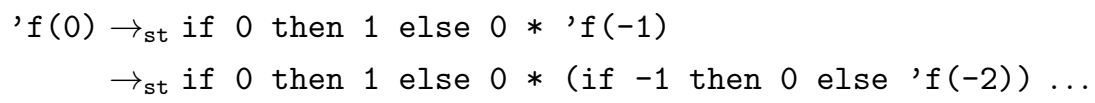

Still, this is not enough when evaluating terms like ' $f($ ' $f(0))$. So, in general, we must ensure not to reduce conditional branches until the condition is solved, no matter if calling by value or by name. To make strategies aware of these precautions while saving code, we will take advantage of strategy parameterization too: we define an additional parameterized module STRAT-EXTENSION that extends a strategy facing function calls st to a strategy xst that applies the reduction step to any program term, but reducing conditions first.

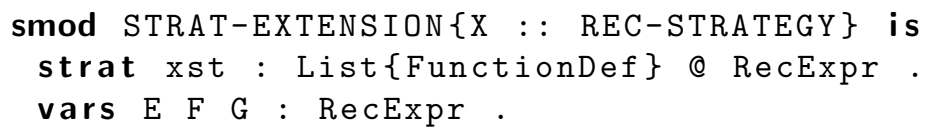




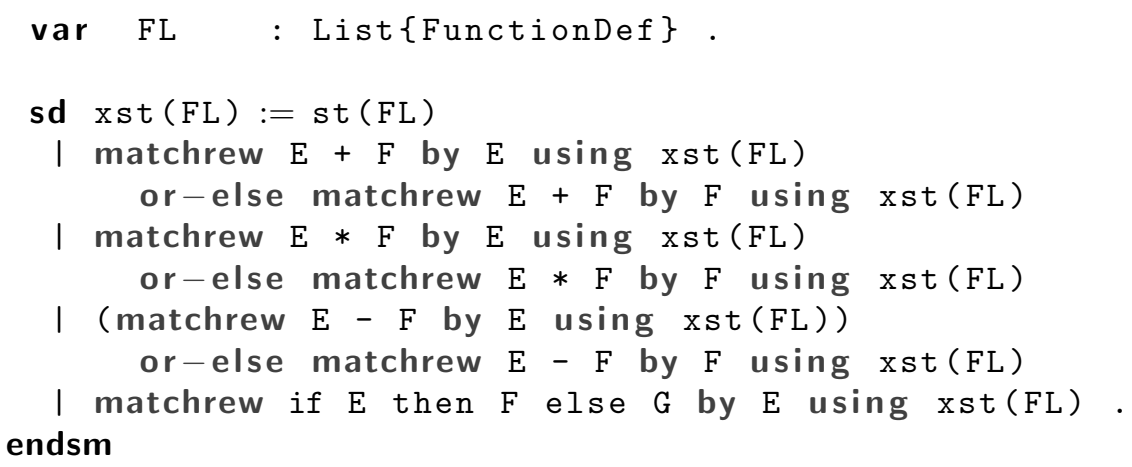

Now, the strategy extension is a reusable component that can be used to define the alternative strategies succinctly, avoiding boilerplate code. First, we instantiate STRAT-EXTENSION with views for byname and byvalue, defined in REC-STRATS, to obtain the extended strategies. Then, we instantiate REC-MAIN with new views from REC-STRATEGY to the instantiated modules, mapping st to the extended strategies. On the contrary, the strategy free applies reduction carelessly and anywhere, so it instantiates REC-MAIN directly.

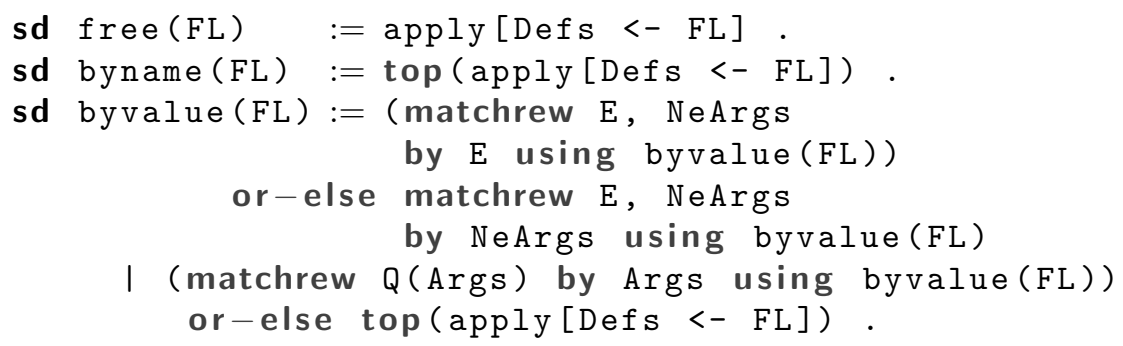

The FL parameter must be filled with the program function definitions. For example, with the Ackermann function defined below we execute

$$
\text { srew } 1+' A(2,3) \text { using reduce (ackermann). }
$$

and obtain 10.

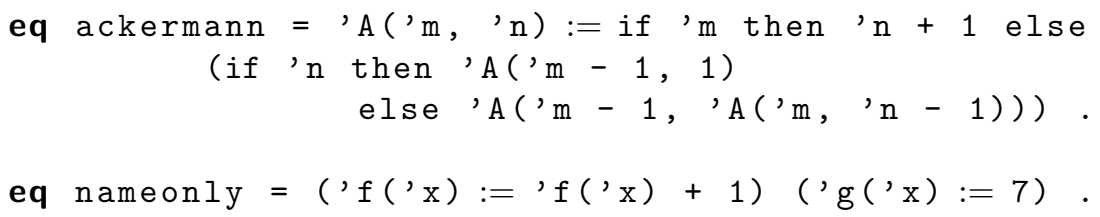

The differences between call by value and call by name are appreciated with the nameonly example, since $g(f(0))$ will be evaluated to 7 by name while it will never finish by value.

\section{Conclusions}

Rewriting strategies are a useful tool in the description of concurrent and logical systems, in accordance with the separation of concerns principle. A stratified 
specification of rules and its global control allows discharging the functional and rule levels of improper complexity, and makes the specified systems more configurable and adaptable. On the other hand, parameterized modules are a basic feature for building complex systems, reusing abstract specifications, and switching between alternative components. Such specifications can be compared in their multiple instances or analyzed parametrically.

The Maude strategy language implementation is now complete, including parameterized strategy modules. In this paper we have seen some examples of parameterized specification with strategies related to algorithms and programming languages. In the future, the combination of strategies and parameterization can be used as a fundamental approach to specify more complex and interesting systems. Also, more elaborated analyses can be done on these, including model checking, whose strategy-aware implementation is currently under development.

\section{References}

1. Andrei, O., Lucanu, D.: Strategy-based proof calculus for membrane systems. In: Roşu, G. (ed.) Proceedings of the Seventh International Workshop on Rewriting Logic and its Applications, WRLA 2008, Budapest, Hungary, March 29-30, 2008. ENTCS, vol. 238(3), pp. 23-43. Elsevier (2009). https://doi.org/10.1016/j.entcs.2009.05.011

2. Borovanský, P., Kirchner, C., Kirchner, H., Ringeissen, C.: Rewriting with strategies in ELAN: A functional semantics. Int. J. Found. Comput. Sci. 12(1), 69-95 (2001). https://doi.org/10.1142/S0129054101000412

3. Bouhoula, A., Jouannaud, J.P., Meseguer, J.: Specification and proof in membership equational logic. Theoretical Computer Science 236(1), 35-132 (2000). https://doi.org/10.1016/S0304-3975(99)00206-6

4. Braga, C., Verdejo, A.: Modular structural operational semantics with strategies. In: van Glabbeek, R., Mosses, P.D. (eds.) Proceedings of the Third Workshop on Structural Operational Semantics, SOS 2006, Bonn, Germany, August 26, 2006. ENTCS, vol. 175(1), pp. 3-17. Elsevier (2007). https://doi.org/10.1016/j.entcs.2006.10.024

5. Bravenboer, M., Kalleberg, K.T., Vermaas, R., Visser, E.: Stratego/XT 0.17. A language and toolset for program transformation. Science of Computer Programming 72(1-2), 52-70 (2008). https://doi.org/10.1016/j.scico.2007.11.003

6. Clavel, M., Durán, F., Eker, S., Lincoln, P., Martí-Oliet, N., Meseguer, J., Talcott, C.: All About Maude-A High-Performance Logical Framework, LNCS, vol. 4350. Springer (2007). https://doi.org/10.1007/978-3-540-71999-1

7. Clavel, M., Durán, F., Eker, S., Lincoln, P., Martí-Oliet, N., Meseguer, J., Talcott, C.: Maude Manual (v2.7.1) (July 2016), http://maude.cs.uiuc.edu/

8. Eker, S., Martí-Oliet, N., Meseguer, J., Verdejo, A.: Deduction, strategies, and rewriting. In: Archer, M., de la Tour, T.B., Muñoz, C. (eds.) Proceedings of the 6th International Workshop on Strategies in Automated Deduction, STRATEGIES 2006, Seattle, WA, USA, August 16, 2006. ENTCS, vol. 174(11), pp. 3-25. Elsevier (2007). https://doi.org/10.1016/j.entcs.2006.03.017

9. Hidalgo-Herrero, M., Verdejo, A., Ortega-Mallén, Y.: Using Maude and its strategies for defining a framework for analyzing Eden semantics. In: Antoy, S. (ed.) Proceedings of the Sixth International Workshop on Reduction Strategies in Rewriting 
and Programming, WRS 2006, Seattle, WA, USA, August 11, 2006. ENTCS, vol. 174(10), pp. 119-137. Elsevier (2007). https://doi.org/10.1016/j.entcs.2007.02.051

10. Martí-Oliet, N., Meseguer, J.: Rewriting Logic as a Logical and Semantic Framework, pp. 1-87. Springer Netherlands (2002). https://doi.org/10.1007/978-94-0170464-9_1

11. Martí-Oliet, N., Meseguer, J., Verdejo, A.: Towards a strategy language for Maude. In: Martí-Oliet, N. (ed.) Proceedings of the Fifth International Workshop on Rewriting Logic and its Applications, WRLA 2004, Barcelona, Spain, March 27-April 4, 2004. ENTCS, vol. 117, pp. 417-441. Elsevier (2004). https://doi.org/10.1016/j.entcs.2004.06.020

12. Martí-Oliet, N., Palomino, M., Verdejo, A.: Strategies and simulations in a semantic framework. Journal of Algorithms 62(3), 95 - 116 (2007). https://doi.org/10.1016/j.jalgor.2007.04.002

13. Meseguer, J.: Conditional rewriting logic as a unified model of concurrency. Theoretical Computer Science 96(1), 73 - 155 (1992). https://doi.org/10.1016/03043975(92)90182-F

14. Murty, K.G.: Linear programming. John Wiley \& Sons, New York (1983)

15. Rubio, R., Martí-Oliet, N., Pita, I., Verdejo, A.: Strategy language for Maude web page, http://maude.sip.ucm.es/strategies

16. Verdejo, A., Martí-Oliet, N.: Basic completion strategies as another application of the Maude strategy language. In: Escobar, S. (ed.) Proceedings 10th International Workshop on Reduction Strategies in Rewriting and Programming, WRS 2011, Novi Sad, Serbia, 29 May 2011. EPTCS, vol. 82, pp. 17-36 (2011). https://doi.org/10.4204/EPTCS.82.2

17. Winskel, G.: The Formal Semantics of Programming Languages. Foundations of Computing, The MIT Press (1993) 